\title{
Consideraciones Sobre la Competencia Comunicativa Oral Profesional Pedagógica en Inglés
}

\section{Considerations About Pedagogical and Professional Oral Communicative Competence in English}

\author{
Julio César Rodríguez Peña ${ }^{1}$ \\ Máster en Ciencias de la Educación (UCPHJLC) y Doctorante en Ciencias Pedagógicas (UCPJLC). Docente de la Universidad de Ciencias Pedagógicas \\ José de la Luz y Caballero y de la Universidad de Ciencias Pedagógicas José de la Luz y Caballero, Holguín (UCPH), Cuba)juliorp@ucp.ho.rimed.cu
}

\begin{abstract}
Alberto Román Medina Betancourt ${ }^{2}$
Doctor en Ciencias Pedagógicas (UCPJLC), Docente Universidad de Ciencias Pedagógicas José de Luz y Caballero, Holguín (UCPH), Cuba) Docente en la Universidad de Ciencias Pedagógicas José de la Luz y Caballero, Holguín (UCPH), Cuba y docente en la Asociación de Pedagogos de Cuba, Instituto Pedagógico Latinoamericano y Caribeño (IPLAC) alberto@ucp.ho.rimed.cu
\end{abstract}

Rafael Lorenzo Martín

Doctor en Ciencias Pedagógicas (UCPJLC) y Máster en Didáctica de la Matemática, Universidad de Ciencias Pedagógicas José de la Luz y Caballero (UCPJLC), Docente de Universidad de Holguín Oscar Lucero Moya (UHOLM), provincia Holguín, Cuba, rlorenzo@ict.uho.edu.cu

Recibido: abril 20 de 2013

Aceptado: mayo 20 de 2013

\section{RESUMEN}

En el artículo se abordó uno de los problemas más importantes y actuales para el proceso de enseñanza-aprendizaje del inglés como lengua extranjera: la formación y desarrollo de la competencia comunicativa oral profesional pedagógica. Para ello, los autores realizaron una sistematización de los elementos esenciales que han dado tratamiento al tema. Investigación descriptiva, desde una posición crítica y dialéctica permite revelar faltantes a resolver por vía científica que dieron lugar a los resultados de un proyecto de investigación orientado a la didáctica específica del Inglés como segunda lengua. Para la fase de diagnóstico-fáctico: observación científica, se encuestaron a 70 estudiantes seleccionados desde un muestreo aleatorio estratificado de la carrera de Licenciatura en Educación, especialidad Lengua Inglesa, que representa un 35,2\% de la población. Una segunda encuesta, se aplicó a 10 profesores de Práctica Integral de la Lengua Ingles y se entrevistaron 20 docentes con experiencia en la Educación Superior Pedagógica El objetivo fundamental radica en el análisis de fundamentos teóricometodológicos sobre las principales categorías pedagógicas de la competencia comunicativa oral profesional pedagógica en inglés, con un enfoque sistémico-comunicativo. Todo lo anterior conforma la novedad de una investigación terminada en la Universidad de Ciencias Pedagógicas de Holguín, en especial en los profesores en formación de inglés.

Palabras clave: Fundamentos Teórico-Metodológicos; Enseñanza-Aprendizaje Del Inglés; Competencia Comunicativa Oral Profesional Pedagógica; Enfoque Sistémico-Comunicativo

\begin{abstract}
The article dealt with one of the most important and updated problems of the teaching-learning process of English as a foreign language: the formation and development of the pedagogical and professional oral communicative competence. To do so, the authors carried out an analysis of different sources which have to do with this theme. Descriptive research, from a position critical and dialectic to reveal missing to resolve through the science that gave rise to the results of a research project aimed at the specific teaching of English as a second language. For the diagnostic - factice phase: scientific observation, surveyed 70 students selected from a random stratified sampling of the Bachelor's degree in education, English language specialty race, representing $35.2 \%$ of the population. A second survey was applied to 10 teachers of comprehensive practice of the English language and interviewed 20 teachers with experience in teaching higher education.The main objective is
\end{abstract}

Profesor (Coordinador de primer año, Categoría Docente Asistente, Máster en Ciencias de la Educación) del Departamento de Lenguas Extranjeras Inglés de la Universidad de Ciencias Pedagógicas de Holguín.

2 Profesor (Jefe de la carrera Licenciatura en Educación, especialidad Lenguas Extranjeras Inglés, Categoría Docente Profesor Titular, Doctor en Ciencias Pedagógicas y Máster en Educación Superior) del Departamento de Lenguas Extranjeras Inglés de la Universidad de Ciencias Pedagógicas de Holguín. 
aimed at the analysis of theoretic-methodological foundations about the main pedagogical categories of the pedagogical and professional oral communicative competence in English, with a systemic-communicative approach. Everything previously stated constitutes the novelty of an investigation concluded in the University of Pedagogical Sciences in Holguín, especially in teachers-to-be of English.

Key words: Theoretic-Methodological Foundations; Teaching-Learning Of English; Formation; Development; Pedagogical And Professional Oral Communicative Competence; Systemic-Communicative Approach.

\section{Introducción}

En el contexto del nuevo modelo de formación de profesores de Inglés en las universidades de Ciencias Pedagógicas de Cuba, en el cual se realizan los tres primeros años en el campus de la Universidad de Ciencias Pedagógicas y completan su formación inicial en condiciones de universalización en una microuniversidad y bajo la guía de un tutor. Los profesores en formación, deben alcanzar los niveles competencia comunicativa en la lengua extranjera que les permita utilizarla como instrumento básico para la dirección del proceso pedagógico centrado en el aprendizaje del inglés como lengua extranjera en cualquier institución educacional.

A través de las diferentes vías establecidas para el control al proceso de formación y evaluación de la calidad de los futuros graduados y de resultados de investigaciones científicas precedentes $(1 ; 2 ; 3 \mathrm{y}$ 4) en el caso particular de la Universidad de Ciencias Pedagógicas de Holguín (Cuba), se ha podido comprobar que los niveles de la competencia comunicativa que se alcanzan no satisfacen las expectativas previstas para continuar la formación de la carrera en los municipios de residencia. Es esencial significar, que existen limitaciones lingüísticas en la lengua extranjera para dirigir el aprendizaje en su actividad durante la práctica preprofesional. En consecuencia, existe la necesidad de resolver tal problema detectado por vía empírica, para elevar los niveles de eficiencia en los futuros profesionales.

La búsqueda realizada en las fuentes teóricas existentes, tanto al nivel internacional, nacional, como territorial, y en particular, las tesis de maestrías y doctorados concluidas, permite afirmar que la habilidad de expresión oral en el inglés como lengua extranjera ha recibido profuso tratamiento ( $5 ; 6$ y 7 ), pero no el necesario y suficiente. En particular, se acentúa la carencia de modelos para la formación de la habilidad desde un enfoque profesional pedagógico para los estudiantes universitarios que se forman como futuros profesores de Inglés.

En correspondencia con lo hasta aquí expuesto, en el artículo se realiza una aproximación teórica a las categorías clave que se relacionan con la temática objeto de tratamiento: La enseñanzaaprendizaje del inglés y el enfoque comunicativo, competencia comunicativa, habilidad de expresión oral en inglés como lengua extranjera, enfoque profesional y 'teacher talk'.

\section{Metodología}

La metodología empleada, para esta investigación descriptiva, mantiene un algoritmo que implica la constante comparación desde lo empírico y la visualización de estados deseados acorde al real y, el supuesto análisis de la argumentación teórica. Lo anterior desde una posición crítica y dialéctica permite revelar faltantes a resolver por vía científica que dieron lugar a los resultados de un proyecto de investigación orientado a la didáctica específica del Inglés como segunda lengua, lo que presupone la génesis y móvil científico de la tesis doctoral del investigador Julio César Rodríguez Peña, titulada: "La competencia comunicativa oral profesional pedagógica en inglés en los estudiantes de la carrera Licenciatura en Educación, especialidad Lenguas Extranjeras Inglés".

Como métodos del nivel teórico, con la finalidad de develar el conocimiento científico a un nivel teórico, se aplicaron: históricológico, inducción- deducción, abstracto a lo concreto, análisissíntesis y el hermenéutico dialéctico; y del nivel empírico, con la finalidad de indagar sobre el proceso de enseñanza-aprendizaje del inglés como lengua extranjera, principalmente en la fase de diagnóstico-fáctico: observación científica. Se encuestaron a 70 estudiantes seleccionados desde un muestreo aleatorio estratificado de la carrera de Licenciatura en Educación, especialidad Lengua Inglesa, que representa un $35,2 \%$ de la población. Una segunda encuesta a 10 profesores de Práctica Integral de la Lengua Inglesa, especialistas en la enseñanza-aprendizaje de esta lengua, de los cuales cuatro ostentan la categoría docente superior y cinco el título académico de Máster o de Especialista; y entrevistas a 20 profesores, la mitad de los cuales coinciden con los encuestados y los restantes son especialistas de vasta experiencia en la Educación Superior Pedagógica, los cuales fueron decisivos para la realización del estudio histórico-lógico.

Este trabajo, se ha concentrado en los campos de la lingüística y de la comunicación para la enseñanza, por lo que los conceptos que aquí se presentan tienen su aplicabilidad directa en el proceso de enseñanza-aprendizaje de lenguas extranjeras. Además la literatura especializada, en libros, artículos y tesis, fue fundamental en la sistematización de los principales aspectos tratados de la enseñanza-aprendizaje del inglés y el enfoque comunicativo, competencia comunicativa, habilidad de expresión oral en inglés como lengua extranjera, enfoque profesional y 'teacher talk'. 


\section{Analisis de los resultados}

Se inició el análisis de este trabajo con la discusión de aspectos puntuales del inglés y el enfoque comunicativo. En el mundo contemporáneo, teniendo en cuenta la situación económica, política y social, y particularmente el desarrollo de la informática y las comunicaciones, se hace imprescindible el dominio de una lengua extranjera.

Asimismo, se conoce que uno de cada tres habitantes del planeta habla inglés, ya sea como resultado de vivir en un país de habla inglesa o por haberlo estudiado en las escuelas de manera institucionalizada. En el primer caso, generalmente se piensa en los hablantes de países como los Estados Unidos de América, Inglaterra y Canadá. Sin embargo, el inglés tiene estatus de lengua oficial en cuarenta y cinco países.

El inglés es un idioma que está presente en las actividades más importantes que se llevan a cabo a escala mundial. Entre estas se encuentran: industriales, científicas, biológicas y proyectos químicos, así como proyectos culturales. Particularmente, en la segunda mitad del Siglo XX, el inglés alcanzó el estatus de lengua internacional de las ciencias y las tecnologías. Como lengua internacional, el inglés se está utilizando de manera cada vez más creciente en contextos no nativos. En consecuencia, hoy nadie en el mundo se cuestiona que esta lengua es la más enseñada, leída y hablada que haya conocido el mundo, pues en los tiempos modernos es la lengua más utilitaria y funcional.

En correspondencia con tales condiciones en la arena internacional, el Sistema Nacional de Educación en Cuba, desde los inicios de la Revolución, le ha dado relevancia al estudio de esta lengua. La enseñanza-aprendizaje del inglés ha pasado a través de diferentes transformaciones a lo largo del discurrir histórico. En estos momentos, en el período que se ha considerado como una nueva revolución educacional, esta tiene vital importancia en el empeño de lograr que todos los ciudadanos alcancen una cultura general integral. Es por ello que, en todos los planes de estudio de las diferentes carreras profesionales, en la Universidad de Ciencias Pedagógicas de Holguín, Cuba se prioriza y se exige la enseñanza-aprendizaje del inglés como lengua extranjera.

En la búsqueda de las regularidades en el proceso de enseñanzaaprendizaje del inglés como idioma extranjero, especialmente en el de la expresión oral, un análisis fue realizado a partir del estudio diagnóstico, mediante la aplicación de diferentes métodos científicos del nivel empírico. El propósito fue determinar el nivel de formación y desarrollo de la habilidad de expresión oral en inglés desde un enfoque profesional pedagógico en los estudiantes de la Especialidad Lenguas Extranjeras Inglés. El análisis del plan de estudios, modelo del profesional, programa y orientaciones metodológicas existentes, la observación directa a clases de inglés en el primer, segundo y tercer años, encuestas y entrevistas a estudiantes y profesores fueron también usadas, para el cumplimiento de los objetivos:
- Determinar las regularidades predominantes al tratar actividades de expresión oral de la lengua inglesa desde un enfoque profesional pedagógico.

- Evaluar el nivel de exigencia de las actividades planificadas para la formación y desarrollo de la habilidad de expresión oral de la lengua inglesa con un enfoque profesional pedagógico.

Para el mejor acercamiento al objeto investigado, se consideraron dos variables principales: expresión oral y, enfoque profesional pedagógico. Dentro de la primera variable, se delimitaron las dimensiones: exactitud, fluidez, lo sociolingüístico, lo estratégico y lo profesional pedagógico. En la exactitud: pronunciación, léxico, gramática, entonación y ritmo, medida por pruebas pedagógicas y la observación científica; en la fluidez: rapidez, organización lógica de las ideas y utilización de claves para la formulación del mensaje. Asimismo, en lo sociolingüístico: conocimiento sobre las normas culturales, estilos y registros lingüísticos, adecuación del texto al mensaje, evidencia de cooperación en la interacción; en lo estratégico: estrategias verbales, estrategias no verbales, claves de inicio, claves de mantenimiento y claves de cierre. En tanto, en lo profesional pedagógico: uso de términos técnicos propios de la especialidad, gestos, expresiones verbales que se utilizan para hacerse comprender y uso de medios complementarios.

Dentro de la variable: Enfoque Profesional Pedagógico, se delimitaron las dimensiones: modo de actuación, lo comunicativo, lo ético profesional, lo psicológico y lo sociológico. En modo de actuación: lógica de actuación profesional y manejo de medios técnicos; en lo comunicativo: evidencia de una comunicación afectiva, modelo comunicativo que se va a transmitir; en la ética profesional: tratamiento a las correcciones de lengua, trato respetuoso y ajuste a las normas de la ética profesional. En consecuencia, en lo psicológico: atención a las particularidades individuales de los estudiantes, distribución de tareas y de responsabilidades de las tareas de acuerdo con necesidades y posibilidades. De tal forma que en lo sociológico: ajuste de las exigencias al contexto social del estudiante y orientación hacia el valor social.

Resultados de la aplicación de instrumentos, De las respuestas obtenidas con la aplicación de los instrumentos, se derivan las siguientes características distintivas:

- Los ejercicios empleados en las clases no son suficientemente variados. Existe una tendencia a realizar los mismos ejercicios que aparecen en el Spectrum y en los cuadernos de trabajo.

- La motivación es un factor importante que a veces es olvidado por los profesores de Inglés, pues las técnicas y procedimientos que utilizan, así como el contenido de los textos no favorecen los niveles motivacionales en los estudiantes.

- No todos los profesores están completamente adiestrados para idear ejercicios desarrolladores de habilidades comunicativas.

- Los profesores consideran que el nivel de los estudiantes es relativamente bajo y que su limitación más grande es la falta 
de motivación; sin embargo, no se responsabilizan con las causas de tal problema ni dominan las estrategias necesarias para elevar los niveles motivacionales hacia la profesión pedagógica.

- En las sesiones metodológicas no se le da el debido seguimiento a los resultados alcanzados por los estudiantes en la formación y desarrollo de la habilidad de expresión oral, siguiendo un enfoque profesional pedagógico.

- No existe una total vinculación de los ejercicios con situaciones similares a las de la vida preprofesional y laboral futura a la que se enfrentarán en años posteriores.

- La mayor parte de los estudiantes reaccionaron correctamente al interactuar con ejercicios que pertenecen a la práctica controlada bajo la guía de los profesores.

- Los estudiantes hicieron sólo ejercicios similares propuestos en el libro de texto y cuaderno de trabajo.

- Existen insuficiencias de adecuación por parte de los estudiantes en las diferentes situaciones comunicativas en un contexto dado, principalmente en el contexto profesional pedagógico.

A partir de la triangulación de los principales resultados de los diferentes métodos aplicados y con el auxilio de un proceso de análisis y síntesis, se pudieron determinar las siguientes regularidades que se constituyen en la argumentación del estado real del objeto investigado:

- La interacción con el proceso de enseñanza-aprendizaje del inglés en el primer, segundo y tercer años de la Especialidad Lenguas Extranjeras Inglés permitió determinar que este proceso es dirigido de manera sistemática por un claustro de profesores que se esfuerza por cumplir los objetivos previstos para el nivel y con estudiantes que en general comprenden la importancia de aprender el inglés como lengua extranjera y lengua que será de vital uso en su vida profesional futura.

- La profundización en los elementos de naturaleza didácticometodológica posibilitó precisar que los profesores de Inglés, aunque conocen que la habilidad objeto de análisis es importante, no le dan la jerarquía requerida en la dirección del proceso de enseñanza-aprendizaje, siguiendo un enfoque profesional pedagógico.

- Del mismo modo, la interacción con los estudiantes como sujetos de investigación, hizo posible determinar que los mismos no tienen la formación de la habilidad de expresión oral en inglés que se requiere para enfrentar con éxito el reto preprofesional y laboral futuro y presentan limitaciones en la formación de la competencia comunicativa en la lengua extranjera, en particular en elementos de la exactitud y la fluidez, así como en la utilización eficiente de las funciones comunicativas características del nivel que estudian.

El modelo profesional y el enfoque comunicativo. Según el modelo del profesional para la carrera de Licenciatura en Educación, especialidad Inglés, el nuevo egresado deberá lograr la formación integral de los adolescentes y jóvenes en el nivel medio y medio superior, que requiere la sociedad cubana actual, poseedores de una cultura general integral con base humanista, que les permita tomar decisiones sobre su vida futura en correspondencia con las necesidades sociales del país. De acuerdo con esta concepción, los nuevos objetivos declarados en los planes de estudios y los programas de las disciplinas establecen que la meta principal del proceso de enseñanza-aprendizaje del inglés es la comunicación.

Enseñar el inglés partiendo del enfoque comunicativo, debe propiciar el desarrollo de las cuatro habilidades comunicativas rectoras del idioma: comprensión auditiva, expresión oral, comprensión lectora y expresión escrita. Estas habilidades deben ser formadas a favor de una apropiada competencia comunicativa. La competencia debe ser vista, en sentido general, desde una primera aproximación a esta categoría en términos de reglas lingüísticas, psicológicas, sociales y necesarias para usar el lenguaje adecuadamente en cada situación y contexto (8); también, como (9) la aptitud de usar el lenguaje apropiadamente en situaciones sociales diversas.

El enfoque comunicativo surgió como resultado de la evolución en la lingüística como ciencia, en particular, la aparición de la lingüística del texto o discursiva, esencialmente bajo las concepciones del relevante lingüista estadounidense Noam Chomsky, a partir de la década del setenta del pasado siglo. Además, como necesidad de satisfacer las nuevas demandas socio-económicas en cuanto al uso de las lenguas extranjeras, en particular el inglés como lengua de uso de alcance internacional, o sea, esta ha alcanzado el estatus de lengua internacional de las ciencias.

En la enseñanza-aprendizaje del inglés en las universidades de ciencias pedagógicas, se necesita aumentar el nivel del idioma desde el enfoque comunicativo del mismo, lo cual se ha visto afectado, así como también el proceso como un todo, debido a la falta de materiales adecuados para satisfacer los requisitos de las clases de expresión oral, y, los materiales que, al mismo tiempo, están en correspondencia con la realidad mundial y la enseñanza de un idioma extranjero en Cuba hoy en día. Prácticamente se ha convertido en una tradición la utilización de la serie Spectrum como material básico para la dirección del aprendizaje del inglés, con sus correspondientes limitaciones desde el punto de vista sociolingüístico y profesional.

Estudios históricos realizados al proceso de enseñanza-aprendizaje de las lenguas extranjeras en Cuba, han permitido precisar la entrada de los primeros indicios del enfoque comunicativo a finales de la década del setenta del pasado siglo. A los efectos de su evolución, resulta interesante significar el realizado por Rivera (10), el cual presenta una periodización del enfoque comunicativo en la cual se refleja el tratamiento a la fluidez y a la exactitud.

Según el referido estudio, la primera corriente en Cuba fue la 'precomunicativa', caracterizada por la estructuración del proceso sobre la base de situaciones, su base lingüística la constituyó el 
estructuralismo de Noam Chomsky. En este período, se comienza a priorizar la fluidez sobre la exactitud. La segunda corriente fue la 'comunicativa pura' basada en el enfoque comunicativo o enfoque nocio-funcional (11; 12 y 13), la cual se caracterizaba por la estructuración del proceso sobre la base de funciones comunicativas, con la base lingüística de la teoría de las funciones de Wilkins, A. D. (11 y 12). Es en este período en el que alcanza la máxima expresión la hiperbolización de la fluidez en detrimento de la exactitud. La tercera corriente es la 'sistémico-comunicativa', la que constituye una versión suavizada del enfoque comunicativo, o sea, un híbrido entre el enfoque estructural y el nocio-funcional y que pone el énfasis en la unidad de la forma, el significado y la función. Con respecto del tratamiento a la exactitud y la fluidez, en este período se da un tratamiento con mayor flexibilidad y sentido dialéctico.

Por su parte, la competencia comunicativa es una de las más tratadas por especialistas en diversos campos. En este trabajo, se limita a los campos de la lingüística y de la comunicación para la enseñanza, por lo que los conceptos que aquí se presentan tienen su aplicabilidad directa en el proceso de enseñanza-aprendizaje de las lenguas extranjeras.

Algunos autores especialistas en la temáticas objeto de análisis en este estudio ( $14 ; 15 ; 16 ; 17 ; 18$ y 19$)$ coinciden en la esencia de los componentes de la competencia comunicativa aportados por Canale y Swain (20). Los mismos consideran a estos como competencias: lingüística, socio-lingüística, discursiva y estratégica, las cuales le permiten al profesor, o a cualquier hablante de la lengua extranjera, comunicarse de forma efectiva y con la corrección necesaria.

Según Chomsky (21), la competencia comunicativa es el conocimiento implícito o explícito del sistema de la lengua que le permite el desarrollo de las habilidades lingüísticas para la producción de una lengua. Para Crystal (22), está constituida por el conocimiento que tiene la persona de las reglas que gobiernan el uso apropiado de la lengua en una situación social dada. Para Hymes (14), la competencia comunicativa incluye el dominio intuitivo que el hablante nativo posee para usar e interpretar la lengua de forma apropiada, en un proceso de interacción y de relación con el contexto social.

El investigador Savignon (16), enfatiza en la naturaleza dinámica de la comunicación y con ella de la competencia comunicativa y refiere que la comunicación ocurre en una variedad infinita de situaciones, por lo que el éxito que se tenga en el desempeño de un rol determinado, depende de la comprensión del contexto y de la experiencia previa sobre una situación similar. Del mismo modo, para él, cobran importancia los gestos, la entonación y la expresión facial en el acto comunicativo, en el que el contexto desempeña su papel.

Es interesante la forma en que Bachman (23), define la competencia para usar la lengua eficientemente y que, para esta investigadora, se identifica con la competencia comunicativa para el profesor de lenguas extranjeras. En su concepción, la competencia comunicativa está compuesta por tres componentes principales: competencia en la lengua, competencia estratégica y mecanismos psicofisiológicos. El primer componente se refiere al conocimiento de la lengua y en ese conocimiento, según Bachman, se encuentran los componentes de la competencia comunicativa expuestos por Hymes (14), Canale y Swain (20) y Canale (24).

En esta concepción de Bachman se hace una distinción entre la competencia organizacional y la competencia pragmática. La primera controla la estructura formal de la lengua, el dominio que se tenga de sus componentes para poder usarlos adecuadamente. La segunda, tiene que ver, con los aspectos funcionales de la lengua, la sensibilidad para manejarla de acuerdo con el contexto y a la función comunicativa, así como al manejo de registros, la sensibilidad lingüística y las referencias culturales.

Según Medina (2), la competencia comunicativa es una configuración de capacidades, conocimientos, habilidades y hábitos lingüísticos y extralingüísticos que se manifiestan durante el acto comunicativo en la lengua extranjera a través del uso apropiado de esta para satisfacer las necesidades comunicativas individuales y colectivas, con ajuste a las normas lingüísticas, sociolingüísticas, discursivas y estratégicas que se requieran; $y$, evidenciando tanto en el contenido como en la forma del mensaje los altos valores morales tanto universales como los del ciudadano cubano.

A partir del análisis realizado, el autor define la competencia comunicativa como una configuración de capacidades, conocimientos, habilidades y hábitos lingüísticos y extralingüísticos que se manifiestan durante el acto comunicativo en la lengua extranjera a través del uso apropiado de ésta como vehículo esencial para conducir la dirección del proceso de enseñanzaaprendizaje, con ajustes a las peculiaridades del idioma para tal empeño, de acuerdo con las crecientes necesidades individuales y colectivas que pueden tener los estudiantes y de las posibilidades del profesor en formación. Sobre esta categoría se significan como rasgos esenciales que se asumen, su carácter contextualizado e integrador.

Enfoque profesional pedagógico Un aspecto muy importante que con frecuencia es olvidado es el enfoque que debe ser seguido en las clases de Práctica Integral del Inglés para estudiantes de primer y segundo años de la carrera: el enfoque profesional pedagógico. Si se sigue correctamente con este enfoque el estudiante deberá adaptar su forma de hablar para comunicarse de una mejor manera con sus interlocutores, es decir, sus estudiantes.

Además este enfoque incluye hablar de una manera más clara, lenta, usando pronunciaciones y discursos simples, reformulando el mismo mensaje varias veces. Entre pedagogos cubanos existe una tendencia a considerar que la profesionalidad está dada por la cualidad de una persona que realiza su trabajo específico con relevante capacidad para cumplir racionalmente sus objetivos, 
proporcionándole una calidad de vida. Esto se fundamenta en el empleo de los principios, métodos, formas, tecnologías y medios que corresponden en cada caso, sobre una elevada preparación (incluyendo la experiencia) para la transformación y producción de nuevos conocimientos y valores para una mejor actuación.

El enfoque pedagógico profesional es pluridimensional e incluye varios factores como: instrucción metodológica, desarrollo de la capacidad para el autodidactismo, dominio pedagógico y curricular del sistema de conocimientos y desarrollo de un pensamiento alternativo.

Sobre la base del análisis expuesto, en el que se han considerado los aportes de diferentes investigadores y la experiencia del autor, se define el enfoque profesional pedagógico como la dirección del sistema de influencias educativas que se ejerce desde los diferentes niveles organizativos, implicados en los procesos sustantivos universitarios, en particular el colectivo de año como eje integrador de la formación profesional pedagógica en las universidades de ciencias pedagógicas. Todo desde el reconocimiento de la profesionalización del proceso de enseñanza-aprendizaje, que prioriza la preparación del estudiante para identificar y resolver los problemas profesionales desde el primer año de la carrera. Además de tener en cuenta un correcto desarrollo de la competencia comunicativa oral para poder enfrentar su accionar preprofesional y laboral con un carácter integral y contextualizado.

El 'teacher talk', en términos generales, se refiere al dominio y uso apropiado del inglés como lengua extranjera objeto de tratamiento; utilizada como un instrumento básico para la dirección del proceso de enseñanza-aprendizaje para contribuir al tránsito hacia niveles superiores y satisfactorios en el uso de habilidades comunicativas: aplicar estrategias comunicativas y registros lingüísticos que se adecuen a las necesidades y posibilidades crecientes de sus estudiantes y a las suyas propias. El profesor de inglés tiene que ofrecer un modelo correcto y preciso, debido a que el mismo constituye la base para la formación lingüística de sus estudiantes en dicha lengua.

El profesor debe adaptar las formas de hablar para poder alcanzar la comunicación con los que los escuchan, o sea, con sus estudiantes. El 'teacher talk' usualmente incluye hablar más clara y lentamente, usar estructuras simples y reformular el mismo mensaje de varias formas. Y de acuerdo con Chaudron (25) 'teacher talk' es el habla del profesor en el aula de clases de lenguas extranjeras e incluye las siguientes modificaciones:

- La velocidad del habla debe ser más lenta que como normalmente ocurre en la comunicación real.

- Las pausas, que deben ser evidencia de la forma en la que el hablante planifica su acto comunicativo y que tienen significado, son posiblemente más frecuentes y más largas.

- La pronunciación tiende a ser exagerada y simplificada.

- Se utiliza el vocabulario más básico necesario para el contenido.

- La cantidad de subordinación es mucho menor.
- Se utilizan más oraciones declarativas que preguntas.

- Los profesores repiten con mucha más frecuencia.

También, el 'teacher talk' se define como el habla que utilizan los profesores y en la que generalmente se modifican las cuatro áreas: el léxico, la sintaxis, la morfología y el discurso. Sin embargo, se ha podido comprobar que las diferentes modificaciones que resultan del 'teacher talk', comparadas con otros dominios típicos de naturaleza sociolingüística no están suficientemente sistematizadas ni expandidas, y tienden a desaparecer a medida que el curso avanza y los estudiantes de lenguas extranjeras elevan sus niveles de competencia en la lengua extranjera (26).

El autor de este artículo, consideran que el 'teacher talk' es de crucial importancia, no solo para la organización y dirección del proceso de enseñanza de la lengua extranjera, sino y sobre todo, para el proceso de aprendizaje. Este elemento es importante para la organización y dirección de las clases de lengua extranjera porque es a través de la lengua que los profesores de inglés triunfan o fracasan en la implementación de sus planes para la enseñanza. Mientras que en términos de aprendizaje el 'teacher talk' es probablemente la mayor fuente de comprensibilidad de la información de entrada en la lengua extranjera (input) que el estudiante recibe.

A pesar de las notables carencias existentes en relación con el 'teacher talk', las pesquisas realizadas por los autores permiten afirmar que en la literatura especializada se encuentran algunas referencias a los elementos de naturaleza lingüística relacionadas con este importante aspecto de las Didáctica Especial de las Lenguas Extranjeras. Sin embargo, no se encontró ninguna referencia a los elementos de naturaleza extralingüística.

La competencia comunicativa oral profesional pedagógica, a decir de Rodríguez (27), se define en una primera aproximación como una configuración de capacidades, conocimientos, habilidades y hábitos lingüísticos y extralingüísticos que se manifiestan durante el acto comunicativo en la lengua extranjera a través del uso apropiado de esta como vehículo esencial para conducir la dirección del proceso de enseñanza - aprendizaje. Y se desarrolla, dentro de un proceso de organizacióny dirección general que sustenta el sistema de influencias educativas del colectivo de año como eje integrador de la formación profesional pedagógica en las universidades de ciencias pedagógicas. Se da, desde el reconocimiento de la profesionalización del proceso de enseñanza - aprendizaje que priorice la preparación del estudiante para identificar y resolver los problemas profesionales desde el primer año de la carrera. Para lo cual hay que tener en cuenta una correcta formación comunicativa para poder enfrentar su accionar preprofesional y laboral con un carácter integral y contextualizado.

\section{Conclusiones}

A modo de conclusiones se significa:

- El papel que desempeña el uso del inglés en la época contemporánea, como resultado del desarrollo científico-técnico, 
en particular de la informática y las comunicaciones, así como las condiciones existentes en la arena internacional en lo económico, político y social; obliga a la búsqueda de enfoques, métodos, técnicas y procedimientos que posibiliten elevar los niveles de eficiencia en el aprendizaje de esta, la lengua internacional de las ciencias y las tecnologías, la más expandido uso y las más utilitaria que ha conocido la historia de la humanidad.

- En el proceso de enseñanza-aprendizaje del inglés como idioma extranjero, el enfoque comunicativo, en particular en estos momentos su fase sistémico-comunicativa, se presenta como el sustento teórico-metodológico pertinente para el logro de un profesional competente acorde a las demandas actuales contempladas en el Sistema Nacional de Educación y la sociedad cubana actual.

- $\quad$ Para el logro de un profesor de Inglés competente desde el punto de vista comunicativo oral y profesional pedagógico, se requiere, de manera priorizada, la preparación del personal docente encargado de formar y desarrollar los niveles de competencia comunicativa oral en inglés como lengua extranjera en los diferentes niveles educaciones, así como el adecuado tratamiento al enfoque profesional pedagógico de dicha competencia.

- La habilidad de expresión oral es la habilidad comunicativa rectora en la mayoría de los cursos de inglés como lengua extranjera en general y en particular en la formación de los futuros profesores de inglés. Su formación tiene la complejidad, no solo las que les son inherentes al sistema oral de la lengua inglesa, sino que se constituye en contenido y forma en la dirección del proceso de enseñanza - aprendizaje.

- A través de investigaciones realizadas, se ha demostrado que precisa de códigos específicos para un uso eficiente de la lengua oral en la organización y dirección del proceso enseñanza-aprendizaje. En consecuencia, se asume el enfoque profesional pedagógico, que, en el caso particular la formación de los profesores de Inglés, se abordan y elaboran bajo la categoría 'teacher talk'. Entendida esta como el dominio y uso apropiado del inglés como lengua extranjera; utilizándola como un instrumento básico para la dirección del proceso de enseñanza-aprendizaje y contribuyendo al tránsito hacia niveles superiores y satisfactorios en el uso de habilidades comunicativas.

- Significa el estar aplicando estrategias comunicativas y registros lingüísticos que se adecuen a las necesidades y posibilidades crecientes de sus estudiantes y a las suyas propias; compuestas estas no solo por los elementos de naturaleza lingüística, sino también y sobre todo, por los de naturaleza extraverbal.

\section{Referencias}

Rodríguez Deveza, Rafael A. (2005). Concepción Teórico-Metodológica para el Diagnóstico-Formación de las Generalizaciones Gramaticales en la Carrera Licenciatura en Educación, Especialidad de Lengua Inglesa. Tesis en Opción al Grado Científico de Doctor en Ciencias Pedagógicas. Centro de Estudio de Educación Superior “José de la Luz y Caballero”, Holguín.

Medina Betancourt, Alberto R. (2006). Didáctica de los Idiomas con Enfoque de Competencias. Ediciones Cepedid. Impreso y hecho en Colombia. 121 p.

Cruz López, H. (2008). Una Concepción Teórica para evaluar la Calidad de la Formación Inicial del Profesional en la Carrera Licenciatura en Educación Especialidad Lenguas Extranjeras Inglés. Tesis en opción al Grado Científico de Doctor en Ciencias Pedagógicas. Centro de Estudio de Educación Superior “José de la Luz y Caballero". Holguín.

Velázquez, M. (2010). La educación valoral en la disciplina Historia de la cultura de los pueblos de habla inglesa. Trabajo Final en Opción al Título Académico de Máster en Ciencias de la Educación en la Mención de Preuniversitario. Material Docente. Holguín.

Rodríguez Peña, Julio C. (2008). Sistema de tareas de aprendizaje para posibilitar la formación y desarrollo de la habilidad de expresión oral en inglés en los estudiantes de décimo grado del IPVCP “Lucía Íñiguez Landín”. Trabajo Final en Opción al Título Académico de Máster en Ciencias de la Educación en la Mención de Preuniversitario. Material Docente. Holguín.

Fuentes, C. (2008). Metodología para la estimulación de la comunicación oral a través de la incorporación del léxico especializado en la educación preuniversitaria diversificada. Trabajo Final en Opción al Título Académico de Máster en Ciencias de la Educación en la Mención de Preuniversitario. Material Docente. Holguín.

Medina Betancourt, Alberto R. (2008). Optimización del Proceso de Enseñanza-aprendizaje del Inglés en Preuniversitario. Proyecto de Investigación. UCP “José de la Luz y Caballero”. Holguín. Cuba.

Giovannini, A. et.al. (1996). Profesor en Acción. Edelsa. Grupo didascalia. S.A. Madrid. 524 p.

Cassary, D. et. al. (1998). Enseñar Lengua. Editorial Grio. Barcelona. España. 240 p.

Rivera Pérez, Santiago Jorge (2001): Modelo Teórico de la enseñanza sistémico-comunicativa para el desarrollo de la habilidad de comprensión de lectura en inglés en el nivel medio (superior). Tesis Doctoral. ICCP. La Habana. Cuba. 
Wilkins, D. A. (1972). The linguistic and situational content of the common core in a unit/credit system. Strasbourg, France: Council of Europe.

Wilkins, D. A. (1976). National syllabuses. Oxford: Oxford University Press.

Van Ek, J. (1975), The threshold level. Strasbourg, France: Council of Europe.

Hymes, D. (1972). On Communicative Competence. Penguin. Editorial Ariel, Barcelona.

Savignon, S. (1983). Communicative competence: Theory and classroom practice. Reading, MA: Addison-Wesley.

(1990). Communicative language teaching: Definitions and directions. In J. E. Alatis (Ed.), Georgetown University Round Table on Languages and Linguistics, 1990. Washington, DC: Georgetown University Press.

(2002). Communicative language teaching: Linguistic theory and classroom practice. In S. Savignon (Ed.), Interpreting communicative language teaching. New Haven, CT: Yale University Press.

Finnochiaro, M. (1989). The Functional-Notional Approach: From Theory to Practice. Edición Revolucionaria. 235 p.

Richards, Jack C. y Charles Lockhart. (1995). Reflective Teaching in Second Language Classroom. Cambridge University Press.
Canale, M. y M. Swain (1980). The Theoretical bases of communicative approaches to second language. (Trad. E. Ferreiro), en Teoría y Práctica de la lectura y la escritura, Síntesis 9, Madrid.

Chomsky, N. (1965). Aspects of the theory of syntax. Cambridge, MA: MIT Press.

Crystal, D. (1999). Language BLANK literature: from conjunction to preposition. English Today 15, 13-21.

Bachman, L .F. (1981). Formative evaluation in specific purpose program development. In R. Mackay \& J. Palmer (eds.), Language for specific purposes, pp. 106-116. Rowley, Mass. : Newbury House.

Canale, M. (1983). On some dimension of language proficiency. In J. Oller (Ed.), Issues in language testing research (pp. 333-342). Rowley, MA: Newbury House.

Chaudron, C. (1985). Intake: On models and methods for discovering learners' processing of input. Studies in Second Language Acquisition, 7, 1-14.

Kumaravadivelu, B. (2006). Understanding Language Teaching. From Method to Postmethod. Lawrence Erlbaum Associates, Publishers Mahwah, New Jersey London.

Rodríguez Peña, Julio C. (2010): Consideraciones teóricas sobre la expresión oral profesional pedagógica en inglés. Revista Electrónica “Ciencias Holguín” ISSN: 1027-2127 http:// www.ciencias.holguin.cu. En el número 4. Revista indexada en Latindex y en Bases de Datos Cubaciencias. 